VOLUME 26 (2019) 122-130

DOI: $10.24330 /$ ieja.587009

\title{
BIPARTITE GRAPHS AND THE STRUCTURE OF FINITE-DIMENSIONAL SEMISIMPLE LEIBNIZ ALGEBRAS
}

\author{
Rustam Turdibaev \\ Received: 6 December 2018; Revised: 24 February 2019; Accepted: 28 February 2019 \\ Communicated by Burcu Üngör
}

\begin{abstract}
Given a finite connected bipartite graph, finite-dimensional indecomposable semisimple Leibniz algebras are constructed. Furthermore, any finite-dimensional indecomposable semisimple Leibniz algebra admits a similar construction.
\end{abstract}

Mathematics Subject Classification (2010): 17A32

Keywords: Semisimple Leibniz algebra, connected bipartite graph

\section{Introduction}

The finite-dimensional simple Lie algebras over an algebraically closed field of characteristic zero are classified and it is a classical result that a finite-dimensional semisimple Lie algebra is a direct sum of simple Lie subalgebras (see [6]). A finitedimensional module over a semisimple Lie algebra due to Weyl's theorem is completely reducible into a direct sum of simple submodules. Furthermore, a Lie algebra admits a Levi decomposition - a semi-direct sum of a semisimple subalgebra and a maximal solvable ideal.

In this paper a "non-commutative" generalization of Lie algebras, introduced by Bloh ([3]) and later by Loday ([9],[10]) - the so-called Leibniz algebras are studied. Although the classical simplicity for Leibniz algebras implies that it is a Lie algebra, a modified definition of the simplicity was introduced in [5] and has been in use in the various papers on the structure theory of Leibniz algebras. Generalization of semisimplicity for Leibniz algebras draws a parallel with semisimple Lie algebras, which is the main focus of the current work. However, it is well-known that a semisimple Leibniz algebra is not in general a direct sum of simple Leibniz algebras, and the question on the structure of semisimple Leibniz algebras has been open. Recently, in [1, Theorem 3.5] the authors establish the description of the finite-dimensional indecomposable semisimple non-Lie Leibniz algebra using a graph, whose vertexes are the simple Lie subalgebras of the liezation of the Leibniz algebra. 
Motivated by the results of [1], the goal of this work is to shed light on how finitedimensional semisimple Leibniz algebras are built. Turns out, the structure of a finite-dimensional semisimple Leibniz algebra is more clear if instead of a graph (cf [1]), one uses a bipartite graph. The main results are presented in the last section, consisting of the description of semisimple Leibniz algebras from [1] with different proofs and a new construction of finite-dimensional semisimple Leibniz algebras using the bipartition of an associated graph.

\section{Preliminaries}

In the following section necessary definitions and results on Lie algebra and representation theory is given. Connection of Leibniz algebra with Lie algebra and its modules is provided. One of the main ingredients in this work, an analogue of Levi's theorem for Leibniz algebra is obtained directly from the results of $\mathrm{T}$. Pirashvili [12] in Subsection 2.2.

\subsection{Lie algebras and Leibniz algebras.}

Definition 2.1. An algebra $\mathfrak{g}$ over $\mathbb{K}$ is called a Lie algebra over $\mathbb{K}$ if its multiplication $([-,-])$ satisfies the identities:

(1) $[x, x]=0$

(2) $[x,[y, z]]+[y,[z, x]]+[z,[x, y]]=0$ for all $x, y, z \in \mathfrak{g}$ (Jacobi identity).

Definition 2.2. A (left) $\mathfrak{g}$-module is vector space $M$ together with a map $\mathfrak{g} \otimes M \rightarrow$ $M, x \otimes m \mapsto x . m$ such that for all $x, y \in \mathfrak{g}$ and all $m \in M$ we have

$$
[x, y] . m=x \cdot(y \cdot m)-y \cdot(x \cdot m) .
$$

Given a left $\mathfrak{g}$-module action on $M$, one can construct a right action by setting $m . x=:-x . m$ and it satisfies the identity

$$
m \cdot[x, y]=(m \cdot x) \cdot y-(m \cdot y) \cdot x .
$$

Left and right actions induce Lie algebra structure on $M \oplus \mathfrak{g}$, where $M$ becomes an abelian ideal and $\mathfrak{g}$ is a subalgebra. If one has the right action of $\mathfrak{g}$ on $M$ and sets the left action to be zero, this induces a new type of a product that generalizes the Lie bracket on $\mathfrak{g}$ given in the following.

Example 2.3. ([7]) Let $\mathfrak{g}$ be a Lie algebra and $M$ be a $\mathfrak{g}$-module. Consider $L=$ $\mathfrak{g} \oplus M$ with a bracket $\left[\left(g_{1}, m_{1}\right),\left(g_{2}, m_{2}\right)\right]:=\left(\left[g_{1}, g_{2}\right],-g_{2} . m_{1}\right)$. Then

$$
[[x, y], z]=[[x, z], y]+[x,[y, z]]
$$


holds for any $x, y, z \in L$ and this algebra is not a Lie algebra if the action of $\mathfrak{g}$ on $M$ is not trivial.

A similar construction is given in [5].

Definition 2.4. A vector space $L$ with a bracket that satisfies identity (1) is called a Leibniz algebra.

The Leibniz algebra of Example 2.3 is denoted by $\mathfrak{g} \ltimes M$.

For a Leibniz algebra $L$ set $I=\operatorname{Span}\langle[x, x] \mid x \in L\rangle$. Then $I$ is a proper ideal of $L$ and $[L, I]=\{0\}$. If $I=\{0\}$ then $L$ is a Lie algebra (and the converse is also true). For a non-Lie Leibniz algebra the ideal $I$ is always non-trivial and the following notion is well-defined.

Definition 2.5. For a Leibniz algebra $L$ the quotient algebra $\mathfrak{g}_{L}:=L / I$ is a Lie algebra which is called the liezation of $L$.

There is the following short exact sequence and the epimorphism $f$ is universal in the sense that a Leibniz algebra homomorphism from $L$ to any Lie algebra $\mathfrak{g}$ factors through $f$ :

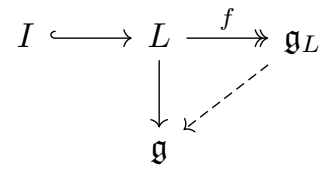

Note that, due to $I$ annihilating the Leibniz algebra whenever multiplied from the right, $I$ admits a structure of a right Lie algebra $\mathfrak{g}_{L}$-module with the well-defined action $i . g=[i, s(g)]$, where $s: \mathfrak{g}_{L} \rightarrow L$ is a linear section.

Definition 2.6. An algebra $A$ is called decomposable if $A=A_{1} \oplus A_{2}$ for some proper ideals. An algebra without this property is called indecomposable.

Any finite-dimensional algebra is either indecomposable or is a finite direct sum of indecomposable algebras. A Lie algebra with no non-trivial ideals is called simple. Simple Lie algebras are indecomposable, while the converse is not necessarily true. A Leibniz algebra with only one non-trivial ideal $I$, so-called simple Leibniz algebra, is indecomposable.

2.2. Levi's Theorem for Leibniz algebras. Similarly, as in Lie algebra theory, the following notions transfer to the case of Leibniz algebra.

Definition 2.7. An ideal $S$ of a Leibniz algebra $L$ is called solvable if $S^{[k]}=\{0\}$ for some integer $k$, where $S^{[0]}=S, S^{[m+1]}=\left[S^{[m]}, S^{[m]}\right]$. The maximum solvable ideal is called solvable radical and is denoted by $\operatorname{Rad}(\mathfrak{g})$. 
A Lie algebra $\mathfrak{g}$ is called semisimple if $\operatorname{Rad}(\mathfrak{g})=\{0\}$. There is a well-known Levi-Malcev decomposition of a finite-dimensional Lie algebra $\mathfrak{g}$ as a semidirect sum of a semisimple subalgebra $\mathfrak{s}$ and the solvable radical, so that $\mathfrak{g}=\mathfrak{s} \ltimes \operatorname{Rad}(\mathfrak{g})$. For Leibniz algebras similar result was proved by D. Barnes [2] in 2011. Note that, the same result is implicit from [12, Proposition 2.4] given below.

Proposition 2.8. Let $\phi: L \rightarrow \mathfrak{g}$ be an epimorphism from an arbitrary finitedimensional Leibniz algebra $L$ to a semisimple Lie algebra $\mathfrak{g}$. Then $\phi$ admits a section.

Indeed, consider a finite-dimensional Leibniz algebra $L$ and apply Levi-Malcev decomposition to its liezation $\mathfrak{g}_{L}$. Applying Proposition 2.8 for an epimorphism $g \circ f$ one obtains a section:

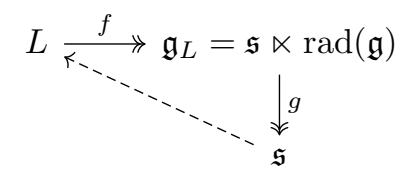

Clearly, the kernel of the epimorphism $g \circ f$ is $\operatorname{Rad}(L)$ and we have an analogue of Levi decomposition for Leibniz algebra $L \cong \mathfrak{s} \ltimes \operatorname{Rad}(L)$.

It is remarkable, that much earlier than the authors mentioned above, A. Bloh established Levi theorem for Leibniz algebras in [4, Theorem 11] and the statement above is also formulated in the following equivalent form [4, Theorem 12]:

Theorem 2.9. Any extension of a semisimple Lie algebra in the class of Leibniz algebras is trivial.

The Malcev part of the theorem is not true in general for the case of Leibniz algebras as shown in [2]. In some cases, conjugacy of Levi subalgebras is possible (see [8] and [11]).

Definition 2.10. A Leibniz algebra $L$ is called semisimple if its liezation $\mathfrak{g}_{L}$ is a semisimple Lie algebra.

Note that, from Levi's decomposition it follows that $L$ is a semisimple Leibniz algebra if and only if $\operatorname{Rad}(L)=I$. Furthermore, there is the following.

Corollary 2.11. Let $L$ be a finite-dimensional semisimple Leibniz algebra. Then $L \cong \mathfrak{g}_{L} \ltimes I$, where $\mathfrak{g}_{L}$ is a semisimple Lie algebra (liezation of $L$ ).

Since $I$ is a $\mathfrak{g}_{L}$-module, and over a semisimple Lie algebra by Weyl's semisimplicity $I$ decomposes into a direct sum of simple $\mathfrak{g}_{L}$-submodules, one obtains

$$
L=\left(\oplus_{i=1}^{m} \mathfrak{g}_{i}\right) \ltimes\left(\oplus_{k=1}^{n} I_{k}\right)
$$


where $\mathfrak{g}_{i}$ 's are simple Lie algebras and $I_{k}$ 's are simple $\oplus_{i=1}^{m} \mathfrak{g}_{i}$-modules.

Let a semisimple Leibniz algebra $L$ be decomposable, that is $L=L_{1} \oplus \ldots \oplus L_{t}$ and $L_{1}, \ldots, L_{t}$ are indecomposable Leibniz algebras. Obviously, $L_{1}$ is also semisimple and Corollary 2.11 implies $L_{1}=\mathfrak{g}_{1} \ltimes I_{1}$, where $\mathfrak{g}_{1}$ as the liezation of $L_{1}$ must be a subalgebra of $\mathfrak{g}_{L}$. Thus, it is a direct sum of some simple components of $\mathfrak{g}_{L}$. Since $L_{1} \unlhd L, I_{1}$ is a $\mathfrak{g}_{L}$-module and using $I_{1} \subseteq I$ it is a sum of simple $\mathfrak{g}$-submodules of I. This implies that not only $L_{1}$ admits the structure of decomposition (2), but $L_{1}=\left(\oplus_{i \in A} \mathfrak{g}_{i}\right) \ltimes\left(\oplus_{k \in B} I_{k}\right)$ for some $A \subseteq\{1, \ldots, m\}, B \subseteq\{1, \ldots, n\}$. Hence, the study of the structure of a semisimple Leibniz algebra is reduced to the study of an indecomposable semisimple Leibniz algebra.

We use the following result from [13, Theorem 6] on the structure of a simple Lie module over a semisimple Lie algebra:

Theorem 2.12. Let $M$ be a finite-dimensional simple Lie algebra module over a finite-dimensional semisimple Lie algebra $\mathfrak{g}=\oplus_{i=1}^{n} \mathfrak{g}_{i}$. Then $M \cong \otimes_{i=1}^{n} M_{i}$, where $M_{i}$ is a simple $\mathfrak{g}_{i}$-module for all $i=1, \ldots, n$.

\section{Main results}

Proposition 3.1. Let $L$ be a finite-dimensional semisimple Leibniz algebra. Then $\left[I_{k}, \mathfrak{g}_{i}\right]=I_{k}$ or $\{0\}$ for all indexes $i$ and $k$ of the decomposition (2).

Proof. Without loss of generality let us consider $\left[I_{1}, \mathfrak{g}_{1}\right]$. Since $I_{1}$ is a simple $\oplus_{i=1}^{n} \mathfrak{g}_{i}$-module, by Theorem $2.12 I_{1}=\otimes_{i=1}^{n} J_{i}$ for simple $\mathfrak{g}_{i}$-modules $J_{i}$. Note that the action is $\left[I_{1}, \mathfrak{g}_{1}\right]=\left[J_{1} \otimes \cdots \otimes J_{n}, \mathfrak{g}_{1}\right]=\left(J_{1} \otimes \cdots \otimes J_{n}\right) \cdot \mathfrak{g}_{1}=\left(J_{1} \cdot \mathfrak{g}_{1}\right) \otimes J_{2} \otimes \cdots \otimes J_{n}$. Now this is either $\{0\}$ or $J_{1} \otimes \cdots \otimes J_{n}=I_{1}$ since $J_{1}$ is an irreducible $\mathfrak{g}_{1}$-module. Moreover, it is well-known from representation theory of Lie algebras that $\left[\mathfrak{g}_{1}, I_{1}\right]=$ $\{0\}$ if and only if $J_{1}=\mathbb{C}$ (a one-dimensional representation is trivial).

Definition 3.2. ([1]) Let $L$ be a semisimple Leibniz algebra with decomposition (2). Two Lie algebras $\mathfrak{g}_{i}$ and $\mathfrak{g}_{j}$ from decomposition (2) are called adjacent if there exists $k$ such that $\left[I_{k}, \mathfrak{g}_{i}\right]=I_{k}=\left[I_{k}, \mathfrak{g}_{j}\right]$.

It is implicit from this definition that for a semisimple Leibniz algebra $L$ with decomposition (2), a graph $\Gamma$ with vertexes $\left\{\mathfrak{g}_{1}, \ldots, \mathfrak{g}_{m}\right\}$ is built. Two vertexes $\mathfrak{g}_{i}$ and $\mathfrak{g}_{j}$ are connected by an edge if $\left[I_{k}, \mathfrak{g}_{i}\right]=I_{k}=\left[I_{k}, \mathfrak{g}_{j}\right]$ for some $k$. The description of indecomposable semisimple Leibniz algebra is established in [1, Theorem 3.5] and rephrased in terms of connectivity of the graph $\Gamma$ below. 
Theorem 3.3. Let $L$ be an indecomposable semisimple Leibniz algebra with decomposition (2). Then $\left[I_{k}, \oplus_{i=1}^{m} \mathfrak{g}_{i}\right]=I_{k}$ for all $1 \leq k \leq n$ and $\Gamma$ is a connected graph.

Proof. If for some $1 \leq k \leq n$ one has $\left[I_{k}, \mathfrak{g}_{i}\right]=\{0\}$ for all $1 \leq i \leq m$, then $I_{k}$ is a direct summand of $L$ (in fact, it is a contradiction with $I_{k} \subseteq I$ being generated by the squares). Thus, from Proposition 3.1 it follows that $\left[I_{k}, \mathfrak{g}_{i}\right]=I_{k}$ for some $1 \leq i \leq m$ which implies the first part of the statement. If graph $\Gamma$ is disconnected, let $\left\{\mathfrak{g}_{i}\right\}_{i \in A}$ be some connected component of $\Gamma$. Using Proposition 3.1 build a set of indexes $B$, where for $p \in B$ there is some $q \in A$ such that $\left[I_{p}, \mathfrak{g}_{q}\right]=I_{p}$. Then $\left(\oplus_{i \in A} \mathfrak{g}_{i}\right) \ltimes\left(\oplus_{k \in B} I_{k}\right)$ is a direct summand of the Leibniz algebra $L$, which is a contradiction.

Using decomposition (2) consider another associated graph on the vertexes

$$
\left\{\mathfrak{g}_{1}, \ldots, \mathfrak{g}_{m}, I_{1}, \ldots, I_{n}\right\}
$$

in which two vertexes are connected via an edge whenever the bracket of the endpoints is a non-zero set. Note that, due to $\left[I_{p}, I_{q}\right]=\left[\mathfrak{g}_{i}, \mathfrak{g}_{j}\right]=\{0\}$, the only edges are from the set of simple Lie algebras $\left\{\mathfrak{g}_{1}, \ldots, \mathfrak{g}_{m}\right\}$ to the set of simple $\mathfrak{g}_{L}$-modules $\left\{I_{1}, \ldots, I_{n}\right\}$.

Definition 3.4. For a semisimple Leibniz algebra $L$ define its corresponding undirected bipartite graph $\mathrm{B} \Gamma$ using decomposition (2) with bipartition $(\mathcal{I}, \mathcal{G})$, where $\mathcal{I}=\left\{I_{1}, \ldots, I_{n}\right\}, \mathcal{G}=\left\{\mathfrak{g}_{1}, \ldots, \mathfrak{g}_{m}\right\}$ and $I_{k} \mathfrak{g}_{i}$ is an edge if and only if $\left[I_{k}, \mathfrak{g}_{i}\right]=I_{k}$.

Theorem 3.5. A finite-dimensional semisimple Leibniz algebra is indecomposable if and only if the associated bipartite graph $B \Gamma$ is connected.

Proof. Assume that B $\Gamma$ is not connected. Let $\left(\left\{g_{i}\right\}_{i \in A},\left\{I_{k}\right\}_{k \in B}\right)$ be one of the connected components of $\mathrm{B} \Gamma$. Then $\left(\bigoplus_{i \in A} g_{i}\right) \ltimes\left(\bigotimes_{k \in B} I_{k}\right)$ is a direct summand of the Leibniz algebra, thus the algebra is decomposable.

Conversely, if the Leibniz algebra is decomposable, then the corresponding bipartite graph is disconnected.

The following corollary establishes that the converse of Theorem 3.3 is also valid.

Corollary 3.6. For a semisimple Leibniz algebra the following conditions are equivalent:

(i) $\left[I_{k}, \oplus_{i=1}^{m} \mathfrak{g}_{i}\right]=I_{k}$ for all $k=1, \ldots, n$ and graph $\Gamma$ is connected;

(ii) $B \Gamma$ is connected. 
Presented example below show that the first condition of the statement $(i)$ is essential.

Example 3.7. Consider a decomposition $\left(\oplus_{i=1}^{4} \mathfrak{g}_{i}\right) \ltimes\left(I_{1} \oplus I_{2} \oplus I_{3}\right)$ with the non-zero products:

$$
\left[I_{3}, \mathfrak{g}_{1}\right]=\left[I_{3}, \mathfrak{g}_{2}\right]=I_{3} \text { and }\left[I_{2}, \mathfrak{g}_{2}\right]=\left[I_{2}, \mathfrak{g}_{3}\right]=\left[I_{2}, \mathfrak{g}_{4}\right]=I_{2} .
$$

The graphs $\Gamma$ and $\mathrm{B} \Gamma$ corresponding to that decomposition are:
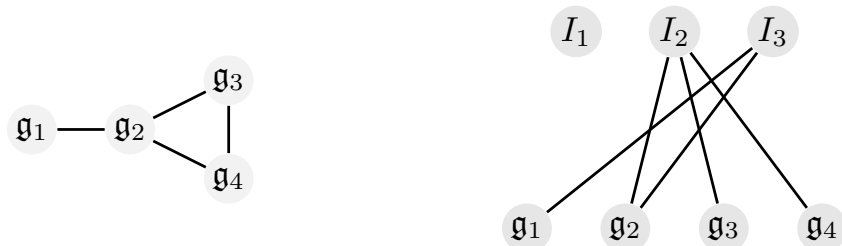

Although $\Gamma$ is connected, but since $\left[I_{1}, \oplus_{i=1}^{4} \mathfrak{g}_{i}\right]=\{0\}$, the Leibniz algebra cannot be indecomposable. In fact, the graph $\mathrm{B} \Gamma$ shows that such Leibniz algebra is not only indecomposable, but does not exist since $I_{1}$ is not being generated by any elements of the Leibniz algebra.

The next statement shows the construction of an indecomposable semisimple Leibniz algebra from any finite connected bipartite graph.

Theorem 3.8. Given a finite and connected bipartite graph, there exists a finitedimensional indecomposable semisimple Leibniz algebra whose corresponding bipartite graph is the given one.

Proof. Let $\mathrm{B} \Gamma$ be a finite, connected bipartite graph with bipartition $(V, W)$. Let us denote formally the vertexes of $V$ by $v_{1}, \ldots, v_{m}$ and the vertexes of $W$ by $w_{1}, \ldots, w_{n}$. The essential $m \times n$ submatrix

$$
A=\left(a_{i j} \mid a_{i j}=1 \text { if } v_{i} w_{j} \text { is an edge and } 0 \text { otherwise }\right)_{1 \leq i \leq m, 1 \leq j \leq n}
$$

of the adjacency matrix of the graph $\Gamma$ contains 1 in every row and in every column since the graph is connected. This submatrix indicates how to build an indecomposable semisimple Leibniz algebra with a corresponding bipartite graph ВГ. Indeed, pick any simple Lie algebra $\mathfrak{g}_{1}, \ldots, \mathfrak{g}_{m}$ and simple $\mathfrak{g}_{i}$-modules $M_{j i}$ for all $1 \leq j \leq n, 1 \leq i \leq m$. Next, define a tensor $\left(\oplus_{i=1}^{m} \mathfrak{g}_{i}\right)$-module $I_{k}=\otimes_{i=1}^{m} J_{k i}$, where $J_{k i}=M_{k i}$ if $a_{k i}=1$ and $J_{k i}=\mathbb{C}$ otherwise. By Theorem 2.12 the $\left(\oplus_{i=1}^{m} \mathfrak{g}_{i}\right)$-module $I_{k}$ is simple for all $1 \leq k \leq n$. Then $L=\left(\oplus_{i=1}^{m} \mathfrak{g}_{i}\right) \ltimes\left(\oplus_{k=1}^{n} I_{k}\right)$ is the Leibniz algebra with corresponding bipartite graph $\mathrm{B} \Gamma$. Note that by Theorem 3.5 it follows that $L$ is indecomposable. 
Example 3.9. Up to an isomorphism there are exactly two connected bipartite graphs with essential submatrix $A$ being a $2 \times 2$ matrix:
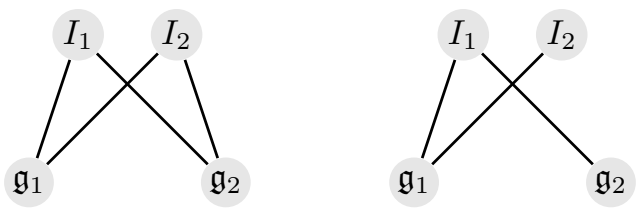

Then by the construction given in the Theorem 3.8 the corresponding indecomposable semisimple Leibniz algebras are the following:

$$
\begin{gathered}
L_{1}=\left(\mathfrak{g}_{1} \oplus \mathfrak{g}_{2}\right) \ltimes\left(\left(J_{11} \otimes J_{12}\right) \oplus\left(J_{21} \otimes J_{22}\right)\right), \\
L_{2}=\left(\mathfrak{g}_{1} \oplus \mathfrak{g}_{2}\right) \ltimes\left(\left(J_{11} \otimes J_{12}\right) \oplus J_{21}\right),
\end{gathered}
$$

where $J_{p q}$ is a simple $\mathfrak{g}_{q}$-module (and for $L_{2}$, $\mathfrak{g}$-module $J_{21} \cong J_{21} \otimes \mathbb{C}$ ). Note that for both $L_{1}$ and $L_{2}$ the graph $\Gamma$ is the same simple connected graph on two vertexes.

In conclusion, the study of the structure of semisimple finite-dimensional Leibniz algebras is complete. Indecomposable such algebras are build by construction from the proof of Theorem 3.8 if one chooses finite-dimensional simple Lie algebras $\mathfrak{g}_{1}, \ldots, \mathfrak{g}_{m}$, well-known finite-dimensional irreducible $\mathfrak{g}_{q}$-modules $J_{p q}$ for all $1 \leq q \leq m, 1 \leq p \leq n$ and a connected bipartite graph with bipartition of vertexes into $m$ and $n$ vertexes.

Acknowledgement. The author would like to thank anonymous referee for suggestions in improving the manuscript.

\section{References}

[1] Sh. Ayupov, K. Kudaybergenov, B. Omirov and K. Zhao, Semisimple Leibniz algebras, their derivations and automorphisms, Linear Multilinear Algebra, (2019), accepted.

[2] D. W. Barnes, On Levi's theorem for Leibniz algebras, Bull. Aust. Math. Soc., 86(2) (2012), 184-185.

[3] A. Bloh, On a generalization of the concept of Lie algebra, Dokl. Akad. Nauk SSSR, 165 (1965), 471-473.

[4] A. Ja. Bloh, A certain generalization of the concept of Lie algebra, Moskov. Gos. Ped. Inst. Ucen. Zap., 375 (1971), 9-20 (in Russian).

[5] A. S. Dzhumadil'daev and S. A. Abdykassymova, Leibniz algebras in characteristic p, C. R. Acad. Sci. Paris Ser. I Math., 332(12) (2001), 1047-1052.

[6] N. Jacobson, Lie Algebras, Interscience Tracts in Pure and Applied Mathematics, No. 10, Interscience Publishers, New York-London, 1962. 
[7] M. K. Kinyon and A. Weinstein, Leibniz algebras, Courant algebroids, and multiplications on reductive homogeneous spaces, Amer. J. Math., 123(3) (2001), 525-550.

[8] K. Kudaybergenov, M. Ladra and B. Omirov, On Levi-Malcev theorem for Leibniz algebras, Linear Multilinear Algebra, 67(7) (2019), 1471-1482.

[9] J.-L. Loday, Cyclic Homology, Grundlehren der Mathematischen Wissenschaften [Fundamental Principles of Mathematical Sciences], 301, SpringerVerlag, Berlin, 1992.

[10] J.-L. Loday, Une version non commutative des algèbres de Lie: les algèbres de Leibniz [A noncommutative version of Lie algebras: the Leibniz algebras], Enseign. Math., 39(2) (1993), 269-293.

[11] G. Mason and G. Yamskulna, Leibniz algebras and Lie algebras, SIGMA Symmetry Integrability Geom. Methods Appl., 9 (2013), 063 (10 pp).

[12] T. Pirashvili, On Leibniz homology, Ann. Inst. Fourier (Grenoble), 44(2) (1994), 401-411.

[13] Z. X. Wan, Lie Algebras, International Series of Monographs in Pure and Applied Mathematics, Vol. 104, Pergamon Press, Oxford-New York-Toronto, Ont., 1975.

\section{Rustam Turdibaev}

Inha University in Tashkent

Ziyolilar 9

100170 Tashkent, Uzbekistan

e-mail: r.turdibaev@inha.uz 